\title{
Memória e gestão estratégica da informação em bibliotecas acadêmicas: mapeamento da produção científica
}

\section{Luciana Bergamo Marques}

Bibliotecária no Serviço de Coleções Especiais da Biblioteca Universitária da Universidade Federal de Santa Catarina (UFSC), mestra em Ciência da Informação pela UFSC e especialista em Administração, Gestão Pública e Políticas Sociais pela UNIESC. Membro estudante do grupo de pesquisa do CNPq "Organização do Conhecimento e Gestão Documental" da UFSC.

bergamota.marques@gmail.com

orcid.org/0000-0002-4640-1470

\section{Cezar Karpinski}

Doutor e mestre em História pela Universidade Federal de Santa Catarina. Bacharel em Filosofia pela Universidade Estadual do Oeste do Paraná. Professor Adjunto IV na Universidade Federal de Santa Catarina, Departamento de Ciência da Informação. É professor do Programa de Pós-Graduação em Ciência da Informação da UFSC e Membro do Grupo de Pesquisa Organização do Conhecimento e Gestão Documental. cezark@hotmail.com orcid.org/0000-0003-2446-0653

\begin{abstract}
Resumo
A proposta desse artigo é analisar, por meio de pesquisa bibliográfica, de abordagem quali-quantitativa, a contribuição da documentação histórica das bibliotecas acadêmicas na gestão estratégica da informação. Foi realizada uma revisão de forma sistemática nas bases de dados Library and Information Science Abstracts, Information Science \& Technology Abstracts, Scopus, Web of Science, Base de Dados Referenciais em Ciência da Informação da Universidade Federal do Paraná e Biblioteca Digital Brasileira de Teses e Dissertações do Instituto Brasileiro de Informação em Ciência e Tecnologia. A estratégia de busca utilizada exigiu a ocorrência simultânea dos termos "memória" ou "história", "biblioteca acadêmica" e "gestão da informação", como descritores ou palavras-chave dos documentos. Foram recuperados 16 artigos, os quais foram categorizados conforme seu objetivo. Desses, nenhum evidenciou o papel da documentação histórica das bibliotecas acadêmicas na gestão estratégica da informação.
\end{abstract}

Palavras-chave: gestão estratégica da informação, memória, biblioteca acadêmica.

Cómo citar este artículo: Marques, L. B., \& Karpinski, C. (2019). Memória e gestão estratégica da informação em bibliotecas acadêmicas: mapeamento da produção científica. Revista Interamericana de Bibliotecología, 42(2), 177-186. doi: 10.17533/udea.rib. $\mathrm{v} 42 \mathrm{n} 2 \mathrm{a} 05$

Recibido: 2017-12-30 / Aceptado: 2019-04-11 


\section{Memory and Information Management in Academic Libraries: Scientific Production}

\begin{abstract}
The purpose of this article is to analyze, through bibliographic research, with a qualitative and quantitative approach, the contribution of historical documentation of academic libraries in the strategic management of information. It was made a bibliographic review on the data bases Library and Information Science Abstracts, Information Science \& Technology Abstracts, Scopus, Web of Science, Base de dados Referenciais em Ciência da Informação of the Universidade Federal do Paraná and Biblioteca Digital Brasileira de Teses e Dissertações of the Instituto Brasileiro de Informação em Ciência e Tecnologia. The search strategy used required the simultaneous occurrence of the terms "memory" or "history", "academic library" and "information management", such as descriptors or keywords. There were retrieved 16 articles that were categorized according to their scope. Of these, none has highlighted the role of historical documentation of academic libraries in the strategic management of information.
\end{abstract}

Keywords: Information management, memory, academic library.

\section{Memoria y gestión estratégica de la información en bibliotecas académicas: mapa de la producción científica}

La propuesta de este artículo es analizar, por medio de investigación bibliográfica, de abordaje cuali-cuantitativo, la contribución de la documentación histórica de las bibliotecas académicas en la gestión estratégica de la información. Se realizó una revisión sistemática en las bases de datos Library and Information Science Abstracts, Information Science \& Technology Abstracts, Scopus, Web of Science, Base de Datos Referenciais en Ciencia de la Información de la Universidad Federal del Paraná y Biblioteca Digital Brasileña de Tesis y Disertaciones del Instituto Brasileño de Información en Ciencia y Tecnología. La estrategia de búsqueda utilizada exigió la coocurrencia de los términos "memoria" o "historia", "biblioteca académica" y "gestión de la información", como descriptores o palabras clave de los documentos. Se recuperaron 16 artículos que fueron categorizados según su alcance. De estos, ninguno ha destacado el papel de la documentación histórica de las bibliotecas académicas en la gestión estratégica de la información.Palabras clave: información, flujo de información, intercambio de información y conocimiento.

Palabras clave: gestión estratégica de la información, memoria, biblioteca académica.

\section{Introdução}

Para que organizações como bibliotecas acadêmicas consigam gerir estrategicamente a informação que geram ou adquirem, antes de tudo, precisam tratá-la diferentemente de outros bens que possuem, pois sua utilidade estratégica aumenta na mesma proporção em que for acessada. Desse modo, os investimentos em informação devem ser eficazes, pois é preciso que os usuários potenciais saibam onde encontrá-la, como acessá-la e como utilizá-la.

Além disso, as bibliotecas acadêmicas não devem considerar apenas as informações recentes, pois a inovação também pode se dar por meio do uso de informações geradas no passado. A memória das organizações tem sido tratada sob diferentes enfoques em variadas áreas do conhecimento (Molina \& V Valentim, 2011). Em todas as abordagens, porém, a memória das organizações acaba convergindo para alguma forma de repositório de conhecimentos tácitos convertidos em explícitos, com o objetivo de disponibilizá-los para aprendizagem e auxílio em processos decisórios (Barbosa, 2013).

Gestão estratégica da informação envolve, portanto, preservar a informação sem torná-la obsoleta. Nesse sentido, a documentação relacionada à memória das instituições representa uma referência à identidade da organização, ao mesmo tempo em que uma fonte de sentidos permanente. Sendo assim, estudar o tratamento que se tem dado aos documentos de memória pode evidenciar meios para melhoria da qualidade e eficiência em produtos e processos de inovação das organizações.

No Brasil, a biblioteca acadêmica é a unidade organizacional responsável pelo armazenamento e disponibilização do conteúdo intelectual da universidade. Além disso, um dos seus objetivos institucionais 
e educar o usuário para o acesso e uso da informação (Lück, 2000). Nesse sentido, o interesse desta pesquisa recai sobre a preservação do conhecimento desenvolvido na biblioteca universitária na prática de sua função e em sua disponibilização a gestores e pesquisadores. Tem-se como pressuposto que o registro documental de suas atividades ao longo do tempo constitui a sua memória e serve, sobremaneira, à gestão estratégica da informação nessas instituições. Coloca-se, então, a seguinte questão de pesquisa: a memória documental está presente na gestão estratégica da informação das bibliotecas acadêmicas?

Buscando responder essa questão, propõe-se, como objetivo geral, analisar, por meio de pesquisa bibliográfica, se e como as bibliotecas acadêmicas têm gerido o seu acervo documental. Entende-se por acervo documental aquele que se relaciona a memória da própria unidade de informação e de acervos especiais.

Com vistas ao cumprimento do objetivo geral foram definidos os seguintes objetivos específicos: a) apontar as correlações entre memória e gestão estratégica da informação; b) avaliar a produção científica quanto à abordagem do conceito de memória na gestão estratégica da informação nas bibliotecas acadêmicas; e c) investigar se há aproveitamento dos documentos de memória na gestão estratégica da informação em bibliotecas acadêmicas.

A motivação para esta investigação partiu da constatação empírica de que, no contexto da literatura sobre bibliotecas, o termo "memória" tem sido utilizado para denotar a formação de coleções sobre história e culturas da comunidade em que a instituição se insere ou da própria instituição (Silva, 2002, Silveira, 2012, Estabel \& Moro, 2014). Desse fato depreende-se que os usos desta categoria e suas aplicações no contexto documental pouco se relacionam com a gestão estratégica da informação.

Percebeu-se também que, em âmbito nacional, a relação dos termos "memória", "bibliotecas acadêmicas" e "gestão estratégica da informação" remetem à curadoria da produção técnico-científica da instituição, encontrando-se o relato da construção de repositórios digitais dessas publicações, como em Santos (2005) e Carmo e Pecegueiro (2011). Por outro lado, há uma grande preocupação dos profissionais de bibliotecas acadêmicas em armazenar e garantir o acesso aos documentos resultantes das atividades de ensino, pesquisa e extensão, por docentes e discentes, o que repercute as ações da Rede Cariniana, pelo Instituto Brasileiro de Informação em Ciência e Tecnologia (IBICT) (Preservação digital, 2012). No entanto, não é a respeito da preservação da produção acadêmica que concerne esta investigação, mas da preservação do registro da práxis em bibliotecas acadêmicas e por essa perspectiva não foram encontrados precedentes, o que corrobora a sua pertinência.

\section{Gestão estratégica da informação e memória documental}

A informação, de um modo geral, pode ser considerada como a representação de pensamentos e acontecimentos que varia conforme o ambiente em que é gerada e o contexto de sua transmissão. A partir dela se estabelecem relacionamentos, produz-se conhecimento e tomam-se decisões. Nas organizações, pode ser aproveitada não apenas no gerenciamento de atividades rotineiras, mas também na projeção de objetivos estratégicos, sendo fundamental para a criação de produtos, serviços e transações inovadoras, desde que entregue significado com eficiência e eficácia (Torres $\&$ Neves, 2008, Camboim, Paiva \& Targino, 2016). Assim, a gestão estratégica da informação ocorre quando há a percepção de oportunidades de melhoria a partir do monitoramento e seleção de informações, do seu tratamento, da sua preservação, comunicação e reutilização. Uma cultura organizacional que fomente esse processo, fazendo uso dos recursos tecnológicos disponíveis, evita que a organização fique à mercê do acaso e agrega valor à informação por promover o aprendizado. Para tanto, um gestor da informação precisa conhecer a cultura do usuário, entendendo como a informação deve ser tratada para que seja acessível e não perca sentido com o passar do tempo (Candido, Valentim \& Contani, 2005).

Para Frade, Nascimento, Tomaél e Neto, (2003), em termos de gestão estratégica da informação, as organizações deveriam concentrar esforços para aumentar a oferta de informações específicas, no momento da tomada de decisão. Já para Wilson, Davenport \& 
Prusak (2004), a informação adquire valor somente quando é transferida. Para estes autores, portanto, a gestão estratégica da informação permite que os indivíduos conversem e escutem o que têm a dizer, pois o conhecimento já existe nas organizações, mas o seu aproveitamento demanda transferência.

As organizações costumam abordar o tema memória quando da criação de produtos que contam uma trajetória, ou valorizem seus acervos, como em repositórios, exposições, livros e documentários (Teixeira, 2002, Silva, 2006, Miranda, 2016, Molina \& Araki, 2016). No entanto, defende-se aqui que a memória das organizações pode ser utilizada como ferramenta de gestão estratégica. Isso porque, na medida em que os documentos registram importantes informações sobre a cultura e os processos organizacionais, o conhecimento que geram é necessário à tomada de decisão no dia-a-dia, ao planejamento de longo prazo e à política de relacionamento com os stakeholders (Lopes, 2004, Gouveia Júnior \& Galindo, 2012). Para tanto, é preciso que profissionais que entendam a natureza desses conteúdos - que, em princípio, já cumpriram o fluxo habitual dentro da organização -, criem repositórios seguindo uma padronização de representação da informação. Dessa forma, agrega-se valor aos documentos e ao processo de gestão, pois facilita o acesso e o uso da informação (Estevão \& Strauhs, 2013).

\section{Metodologia}

Realizou-se uma pesquisa bibliográfica quali-quantitativa, dedicada à análise do conteúdo de publicações científicas que relacionam o conceito de memória à gestão estratégica da informação em bibliotecas acadêmicas. As bases de dados selecionadas foram:

a) Scopus e Web of Science (WoS), por sua ampla abrangência internacional e multidisciplinar;

b) Library, Information Science \& Technology Abstracts (LISTA) e Library and Information Science Abstracts (LISA), pela abrangência internacional e especificidade à área de Ciência da Informação;

c) Biblioteca Digital de Teses e Dissertações do IBICT (BDTD), por reunir a produção brasileira no âmbito da pós-graduação; d) Base de dados Referenciais de Periódicos em Ciência da Informação (BRAPCI), por reunir a produção científica brasileira na área de Ciência da Informação.

Os descritores utilizados para a busca foram "academic library", "academic libraries", "university library", "university libraries", "history", "memory", "information management", "memória", "biblioteca acadêmica", "biblioteca universitária", e "gestão da informação". Uma vez que, em buscas preliminaries nas bases de dados nacionais verificou-se que o termo "biblioteca acadêmica" não retornava resultados quando associado a "gestão da informação", optou-se por incluir nas estratégias da busca sistemática de literatura apenas o termo sinônimo "biblioteca universitária".

O recorte temporal estabelecido se deu no limite do ano final da pesquisa (2017), obedecendo à data inicial de abrangência de cada base. Nesse sentido, é impor tante destacar que os acervos da BDTD e BRAPCI têm como ano de início 1997 e 1972, respectivamente. Esta delimitação ampla teve como objetivo verificar quando e como o tema passou a figurar na comunicação científica. Foram excluídas da análise publicações que não passaram pelo escrutínio de pares, como livros e artigos de opinião, além daquelas em que a leitura de resumo evidenciou não estarem relacionados à memória documental ou a gestão estratégica da informação.

\section{Resultados: apresentação e discussão}

Os documentos recuperados em cada base de dados foram analisados pela leitura de título e resumo e categorizados segundo o seu objetivo. As estratégias de busca utilizadas em cada base de dados estão incluídas nos Quadros 1 a 6, que organizam as referências das publicações, objetivos e descritores, ou palavras - chave. Foram eliminadas da análise as publicações que não eram científicas, resenhas, e-books e trabalhos apresentados em conferências. Assim como os trabalhos que se repetiam em mais de uma base de dados foram considerados apenas uma vez. 
Quadro 1. Publicações recuperadas na base de dados LISA.

Estratégia de busca su("university library" OR "academic library" OR "academic libraries" OR "university libraries") AND su(history OR memory) AND su("information management")

\begin{tabular}{|c|c|c|}
\hline Referência & Objetivo & Descritores \\
\hline $\begin{array}{l}\text { Guard, J. R., Brueggemann, R. F., Fant, W. } \\
\text { K., Hutton, J. J., Kues, J. R., Marine, S. A.,... } \\
\text { \& Schick, L. C. (2004). Integrated Advanced } \\
\text { Information Management Systems: a twenty- } \\
\text { year history at the University of Cincinnati. } \\
\text { Journal of the Medical Library Association, 92(2), } \\
\text { 171-178. }\end{array}$ & $\begin{array}{l}\text { Discutir a história do National Library } \\
\text { of Medicine's Integrated Advanced } \\
\text { Information Management Systems (IAIMS) } \\
\text { na Universidade de Cincinnati e descrever as } \\
\text { metas, métodos e estratégias do programa de } \\
\text { IAIMS atual. }\end{array}$ & $\begin{array}{l}\text { Computerized information work. Medicine. } \\
\text { Management. Integrated systems. Integrated } \\
\text { Academic Information Management Systems. } \\
\text { University libraries. History. USA. Ohio. } \\
\text { Cincinnati University. }\end{array}$ \\
\hline
\end{tabular}

Fonte: elaboração própria.

Quadro 2. Publicações recuperadas na base de dados LISTA.

(academic libraries or university libraries or college libraries) AND (history OR memory) AND information management

\section{Referência}

Turner, A. L. (2006). Committing to memory: a project to publish and preserve California local history digital resources. Journal of Archival Organization, 4(1/2), 11-27.

Marchionini, G. (2014). Libraries of people. Information Studies, 20(3), 143-194.

Line, M. B. (2006). Forty years of library automation: a personal reflection. Program, 40(2), 118-122.

Komlodi, A., Marchionini, G., \& Soergel, D. (2007). Search history support for finding and using information: user interface design recommendations from a user study. Information Processing \& Management, 43(1), 10-29.

Lloyd, A., Harvey, R., Lodge, \& D. (2005). Lost and missing Australian documentary heritage: is there any? Australian Library Journal, 54(3), 288-297.
Investigar o histórico de busca de informações jurídicas por advogados e bibliotecários de direito e fazer recomendações para a melhor usabilidade dos sistemas de informação.

Descrever a evolução das abordagens metodológicas do projeto Lost and Missing, parte do Programa Memória do Mundo, na Universidade Charles Sturt (Austrália), o qual busca identificar património documental perdido.

Fonte: elaboração própria. 
Quadro 3. Publicações recuperadas na base de dados Scopus.

Estratégia de busca
TITLE-ABS-KEY ("university library" OR "academic library" OR "academic libraries" OR "university libraries") AND (history OR memory) AND ("information management") AND LIMIT-TO (SRCTYPE, "j")

\section{Referência}

Objetivo

McRostie, D. (2006). The only constant is change: evolving the library support model for research at the University of Melbourne. Library Management, 37(6/7), 363-372.

Joint, N. (2008). Addled by authentication: recent changes to password systems in British academic libraries: ANTAEUS. Library Review, 57(7), 491-498.

Guise, J. L. (2005). Toward a template for systematic reference and instruction programme analysis. New Library World, $106(1 / 2), 29-42$.

Fonte: elaboração própria.

Quadro 4. Publicações recuperadas na base de dados WoS.

Estratégia de busca

\section{Referência}

McRostie, D. (2016). The only constant is change evolving the library support model for research at the University of Melbourne. Library Management, $37(6 / 7), 363-372$.

Fonte: elaboração própria.

Quadro 5. Publicações recuperadas na base de dados BDTD
Examinar a trajetória e a abordagem utilizada para atender às demandas dos pesquisadores em uma biblioteca acadêmica.

Descrever os princípios gerais subjacentes às mudanças na política e prática de bibliotecas digitais no Reino Unido desde 2006.

Desenvolver um método de análise sistemática de programas de capacitação de usuários em bibliotecas acadêmicas para que os gestores desses serviços possam identificar possíveis áreas de mudança e fazer alterações mais confiantes.
Tópico: (("university library" OR "academic library" OR "academic libraries" OR "university libraries") AND ( history OR memory ) AND ("information management"))

\section{Objetivo}

Relatar a experiência de gestão de serviços voltados às demandas dos usuários na biblioteca universitária da Universidade de Melbourne.
Academic libraries. Reference services. Instructions. Information management.
Data curation. Data management. Digital scholarship. Informatics. Research information management. Skills development.

Academic libraries. Digital libraries. Message authentication. Data security. United Kingdom. 
Quadro 6. Publicações recuperadas na base de dados BRAPCI.

\begin{tabular}{|c|c|c|}
\hline Estratégia de busca & memoria AND biblioteca AND "gestão da inforr & lação" \\
\hline Referência & Objetivo & Descritores \\
\hline $\begin{array}{l}\text { Silva, G. B., Santos, J., Silva, M. L., Galdêncio } \\
\text { de Moura, R. K., \&E Carvalho, M. M. (2013). } \\
\text { A importância da preservação, conservação e } \\
\text { tratamento dos registros arquivísticos da UFRN. } \\
\text { Múltiplos olhares em Ciência da Informação,3(2). 1-12. }\end{array}$ & $\begin{array}{l}\text { Relatar a relevância de uma gestão da } \\
\text { informação fortalecida com o auxilio } \\
\text { dos diferentes profissionais (jornalistas, } \\
\text { bibliotecários e arquivistas), buscando a } \\
\text { responsabilidade em relação ao cuidado devido } \\
\text { ao patrimônio documental do passado e aos } \\
\text { documentos que são produzidos pela UFRN. }\end{array}$ & $\begin{array}{l}\text { Documento. Memória. Tratamento } \\
\text { documental. Gestão da informação. }\end{array}$ \\
\hline $\begin{array}{l}\text { Santos, R. F., \& Silva, E. F. (2013). O bibliotecário } \\
\text { como arquiteto dainformação: os desafios eas novas } \\
\text { abordagens no hodierno contexto. Múltiplos olhares } \\
\text { em Ciência da Informação,3(2). }\end{array}$ & $\begin{array}{l}\text { Identificar a importância do profissional da } \\
\text { informação como Arquiteto da Informação na } \\
\text { estruturação de websites visando à qualidade } \\
\text { da disponibilidade do patrimônio histórico em } \\
\text { meio digital, a fim de possibilitar a garantia do } \\
\text { acesso às gerações futuras. }\end{array}$ & $\begin{array}{l}\text { Gestão da Informação. Memória Documental. } \\
\text { Arquitetura da Informação. }\end{array}$ \\
\hline
\end{tabular}

Fonte: elaboração própria.

A análise dos objetivos dos artigos (coluna CATEGORIA discriminada no Quadro 7) revela a escassa produção sobre a história das bibliotecas acadêmicas, que, ademais, refere-se à história de algum dos seus serviços, como:

a) Curadoria de documentos sobre história e cultura locais ou da produção científica da universidade;

b) Contribuição aos grupos de pesquisa em medicina;

c) Disponibilização do patrimônio histórico em meio digital (websites).

Nas bases de dados internacionais verificaram-se, principalmente, artigos que relatam a constituição da memória das universidades em repositórios institucionais geridos pelas bibliotecas acadêmicas; a trajetória de uma gestão por melhor qualidade e variedade de serviços; e a elaboração e implementação de projetos de digitalização de manuscritos e documentos audiovisuais sobre a região e a comunidade em que se insere.

Quanto às bases de dados nacionais, a busca na BDTD retornou dois resultados referentes à memória e biblioteca acadêmica, todavia, estes tratavam sobre repositório digital de informação técnico-científica institucio- nal e história de serviço de educação de usuários. Por fim, a pesquisa na BRAPCI retornou apenas resultados relativos à gestão da documentação institucional e ao papel do bibliotecário na arquitetura de websites.

Pelo Quadro 7 observa-se que há uma distribuição variada dos resultados ao longo dos últimos anos, havendo o primeiro registro dos termos em conjunto no ano 2002. Nas bases de dados de abrangência internacional, as publicações recuperadas são provenientes de países de língua inglesa, bem como do hemisfério norte, em sua maioria (Estados Unidos da América, Reino Unido e Austrália).

Verifica-se que as bases de dados multidisciplinares contêm menor número de resultados que as bases especializadas. Também é possível afirmar que os periódicos que mais contribuíram com resultados recuperados dedicam-se a aspectos particulares da área, como gestão de bibliotecas e automação. Desse modo, o que se pode observar é que o assunto pesquisado permanece um interesse exclusivo da Ciência da Informação, visto que não foram encontrados registros provenientes de periódicos de outras áreas do conhecimento, exceto pelo que diz respeito a sistemas de informação automatizados. 
Quadro 7. Resumo de categorias, por ano das publicações e base de dados.

\begin{tabular}{|c|c|c|c|c|c|c|}
\hline Categoria & LISA & LISTA & Scopus & Wos & BDTD & BRAPCI \\
\hline $\begin{array}{l}\text { Coleção sobre história local, com a infraestrutura da } \\
\text { biblioteca. }\end{array}$ & & 2006 & & & & 2013 \\
\hline Coleção de memória da instituição. & & & & & & 2016 \\
\hline Estudo de busca e uso da informação. & & 2006, 2007 & & & & \\
\hline Histórico da gestão de serviços de biblioteca acadêmica. & & & $2005,2006,2008$ & 2016 & 2003 & \\
\hline Sistema de gestão de memórias pessoais. & & 2014 & & & & \\
\hline Sistema de gestão de informações na universidade. & 2004,2004 & & & & 2011 & \\
\hline Sistema de recuperação de documentos digitais perdidos. & & 2005 & & & & \\
\hline Papel do bibliotecário na estrutura de websites & & & & & & 2013 \\
\hline Total de publicações & 2 & 5 & 3 & 1 & 2 & 3 \\
\hline
\end{tabular}

Fonte: elaboração própria.

\section{Considerações finais}

A investigação sobre a contribuição dos documentos de memória da biblioteca acadêmica na gestão estratégica da informação, presente na literatura científica, demonstrou que há poucos trabalhos focando a convergência desses conceitos. Como ficou demonstrada pela categorização de escopo das publicações levantadas, a história das bibliotecas acadêmicas tem sido abordada como contextualização dos casos sobre gestão.

As pesquisas sobre a contribuição da memória documental das bibliotecas acadêmicas podem contribuir na prática da gestão de informações, auxiliando no alcance dos objetivos estratégicos da unidade. Há potencial de soluções em gestão estratégica da informação no estudo e tratamento dessa memória, uma vez que os novos conhecimentos perpassam informações geradas no passado e que ainda não foram tratadas para assumirem seu papel no presente e no futuro.

\section{Referências}

1. Barbosa, A. A. (2013). Memória institucional: possibilidade de construção de significados no ambiente organizacional. In Encontro Nacional de História da Mídia, 9. (2013). Ouro Preto (MG). Porto Alegre (RS): ALCAR. Recuperado de http://www.ufrgs.br/alcar/ encontros-nacionais-1/9o-encontro-2013/artigos/ gt-historiografia-da-midia/memoria-institucionalpossibilidade-de-construcao-de-significados-noambiente-organizacional
2. Camboim, L. G., Paiva, S. B. \& Targino, M. G. (2016). Gestão estratégica da informação em coordenações de cursos de graduação de universidades públicas federais. Perspectivas em Gestão e Conhecimento, (1). Recuperado de http://periodicos.ufpb.br/index.php/pgc/article/ view/25158/15482

3. Cândido, C. A., Valentim, M. L. G. P. \& Contani, M. L. (2005). Gestão estratégica da informação: semiótica aplicada ao processo de tomada de decisão. DataGramazero, 6(3). Recuperado de http://www.brapci.ufpr.br/brapci/ $\mathrm{v} / \mathrm{a} / 3073$

4. Carmo, J. R. \& Pecegueiro, C. M. P. (2011). Organização do conhecimento científico na universidade: um estudo de caso. Brazilian Journal of Information Science, 5(2), 97-109. Recuperado de http://www2.marilia.unesp.br/revistas/ index.php/bjis/article/view/1330/2181

5. Estabel, L. B. \& Moro, E. L. S. (2014) Biblioteca: conhecimentose práticas. Porto Alegre: Penso, 2014. Livro eletrônico acessado via matrícula e senha da UFSC em Minha Biblioteca. Recuperado de https://integrada.minhabiblioteca.com. br/A/books/9788565848862

6. Estevão, J. S. B. \& Strauhs, F. R. (2013). Proposta de uma ontologia como modelo de referência no domínio da Memória Organizacional Histórica. Perspectivas em Ciência da Informação, 18(4), 35-53, Recuperado de http://www.scielo. br/pdf/pci/vl8n4/04.pdf

7. Frade, A. C. M. N. et al. (2003). Gestão estratégica da informação: a distribuição da informação e do conhecimento. Informação e Sociedade: Estudos, 13(2), 37-64. Recuperado de http://www.ies.ufpb.br/ojs/index.php/ies/ article/view/90/1557 
8. Gomes, A. L. C. A., \& Nogueira, D. G. P. (2016). Informação e memória no Tribunal de Contas da União (1970-2004). Informação \& Informação, 21(3). 306-326.

9. Gouveia Júnior, M. \& Galindo, M. (2012). Sistemas memoriais como disseminadores da informação. TransInformação, 24(3), 207-217. Recuperado de http:// periodicos.puc-campinas.edu.br/seer/index.php/transinfo/ article/view/1203/1178

10. Guard, J. R., Brueggemann, R. F., Fant, W. K., Hutton, J. J., Kues, J. R., Marine, S. A.,... \& Schick, L. C. (2004). Integrated Advanced Information Management Systems: a twenty-year history at the University of Cincinnati. Journal of the Medical Library Association, 92(2), 171-178.

11. Guizalberth, A. G. (2003). Aprendizagem organizacional em bibliotecas: a experiência do sistema de bibliotecas Unileste (MG) (dissertação mestrado). Belo Horizonte: Universidade Federal de Minas Gerais.

12. Guise, J. L. (2005). Toward a template for systematic reference and instruction programme analysis. New Library World, 106(1/2), 29-42.

13. Joint, N. (2008). Addled by authentication: recent changes to password systems in British academic libraries: ANTAEUS. Library Review, 57(7), 491-498.

14. Komlodi, A., Marchionini, G., \& Soergel, D. (2007). Search history support for finding and using information: user interface design recommendations from a user study. Information Processing \& Management, 43(1), 10-29.

15. Line, M. B. (2006). Forty years of library automation: a personal reflection. Program, 40(2), 118-122.

16. Lück, E. H. et al. (2000). A biblioteca universitária e as diretrizes curriculares do ensino de Graduação. In Seminário Nacional de Bibliotecas Universitárias, 11. Florianópolis: UFSC, 2000. Recuperado de http://snbu.bvs.br/snbu2000/ parallel.html

17. Lopes, U. S. (2004). Arquivos e a organização da gestão documental. Revista ACB: Biblioteconomia em Santa Catarina, 9(1), 113-122. Recuperado de https://revista.acbsc.org.br/ racb/article/view/412/523

18. Lloyd, A., Harvey, R., \& Lodge, D. (2005). Lost and missing Australian documentary heritage: is there any? Australian Library Journal, 54(3), 288-297.

19. Marchionini, G. (2014). Libraries of people. Information Studies, 20(3), 143-194.

20. McRostie, D. (2006). The only constant is change: evolving the library support model for research at the University of Melbourne. Library Management, 37(6/7), 363-372.
21. McGowan, J. J., Overhage, J. M., Barnes, M., \& McDonald, C. J. (2004). Indianapolis I3: the third generation Integrated Advanced Information Management Systems. Journal of the Medical Library Association, 92(2), 179-187.

22. Miranda, R. (2016). A construção do Centro de Documentação e Memória da Faculdade de Educação da UFMG: breve relato. Bibliotecas universitárias: pesquisas, experiências e perspectivas, 3(1). Recuperado de https://seer. ufmg.br/index.php/revistarbu/article/view/1997/1447

23. Molina, L. G. \& Araki, C. (2016). Centros de memória no ambiente digital: em foco a análise de empresas públicas e privadas. Em Questão, 22(1). Recuperado de http://seer.ufrgs. br/index.php/EmQuestao/article/view/58726/36976

24. Molina, L. G. \& Valentim, M. L. P. (2011). Memória organizacional, memória corporativa e memória institucional: discussões conceituais e terminológicas. Revista EDICIC, 1(1), 262-276. Recuperado de http://www. edicic.org/revista/index.php/RevistaEDICIC/article/ view/20/pdf

25. Paiva, M. R. (2011). Compartilhamento da informação e do conhecimento na Universidade Estadual de Maringá: contribuições de um repositório temático institucionalem modae design (dissertação mestrado profissional). Programa de Pós-Graduação em Gestão da Informação. Londrina: Universidade Estadual de Londrina.

26. Preservação digital (2012). Ciência da Informação, 4l(1). Edição especial temática. Recuperado de http://revista. ibict.br/ciinf/issue/view/106/showToc

27. Santos, U. G. (2005). Aspectos gerenciais da Seção Memória da Biblioteca Central da UFBA: interferência no registro das produções acadêmicas. In Encontro Nacional de Ciência da Informação, 6. Salvador. Recuperado de http://www. cinform-anteriores.ufba.br/vi_anais/docs/UbiraciSantos.pdf

28. Silva, H. R. (2002). "Rememoração"/comemoração: as utilizações sociais da memória. Revista Brasileira de História, 22(44). Recuperado de http:// www.scielo.br/readcube/epdf.php?doi=10.1590/ S0102-01882002000200008\&pid=S0102$01882002000200008 \&$ edf_path=rbh/v22n44/14006. pdf\&lang=pt

29. Silva, R. R. G. (2006). Procedimentos básicos de seleção de documentos para conversão digital: elementos de atualização profissional em setores de duas instituições públicas federais. Perspectivas em Ciência da Informação, ll(3), 433-444. Recuperado de http://portaldeperiodicos.eci. ufmg.br/index.php/pci/article/view/56/73

30. Silva, G. B., Santos, J., Silva, M. L., Galdêncio de Moura, R. K., \& Carvalho, M. M. (2013). A importância da preservação, 
conservação e tratamento dos registros arquivísticos da UFRN. Múltiplos olhares em Ciência da Informação, 3(2). 1-12.

31. Silveira, F. J. N. (2010). Biblioteca, memória e identidade social. Perspectivas em Ciência da Informação, 15(3), 67-86. Recuperado de http://www.scielo.br/pdf/pci/vl5n3/05.pdf

32. Santos, R. F., \& Silva, E. F. (2013). O bibliotecário como arquiteto da informação: os desafios e as novas abordagens no hodierno contexto. Múltiplos olhares em Ciência da Informação, 3(2).

33. Teixeira, W. A. M. (2002). Ações de conservação e preservação da memória no contexto digital. TransInformação, 14(2), 179-181. Recuperado de http:// periodicos.puc-campinas.edu.br/seer/index.php/transinfo/ article/view/1500/1474

34. Torres, R. F. \& Neves, J. T. R. (2008). Gestão estratégica da informação: estudo de caso em uma prestadora de serviços de tecnologia da informação. DataGramazero, 9(1). Recuperado de http://www.brapci.ufpr.br/brapci/v/a/4837

35. Turner, A. L. (2006). Committing to memory: a project to publish and preserve California local history digital resources. Journal of Archival Organization, 4(1/2), 11-27.

36. Wilson, N., J. H., Davenport, T. H. \& Prusak, L. (2004). ¿Quién le aporta las buenas ideas y cómo responde usted? Madrid, ES: Deusto. Livro eletrônico acessado via reconhecimento de IP. Recuperado de http://www.ebrary.com 